\title{
Effect of the sample topology on superconducting critical parameters
}

\author{
V. V. Moshchalkov ${ }^{1}$, L. Gielen ${ }^{1}$, C. Strunk ${ }^{1}$, R. Jonckheere ${ }^{2}$, X. Qiu ${ }^{1}$, C. Van Haesendonck ${ }^{1}$ \& \\ Y. Bruynseraede ${ }^{1}$
}

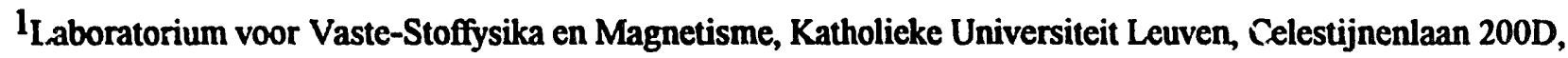
3001 Leuven, Belgium

2Interuniversity Microelectronics Center, Kapeldreef 75, 3001 Leuven, Belgium

The magnetic field $(H)$ - temperature $(T)$ phase boundary has been measured for mesoscopic superconducting samples of different topology (lines, loops and dots). Both the quantization effects and the slope of the $H_{C}(T)$ line are fully governed by the confinement geometry. As a result, "quantum design" of superconducting critical parameters of mesoscopic systems becomes possible.

\section{INTRODUCTION}

The superconducting state can be suppressed by applying a magnetic ficid $H$ or increasing the temperature $T$. It is general? helieved that the phase boundary line separating the superconducting from the normal state in the $\tilde{h}-T$ plane is mainly determined by the material itself. This is indeed true for bulk samples, where the surface to volume ratio is quite small. In mesoscopic superconducting samples, however, this ratio is very large and the nucleation of the superconducting state is strongly dependent upon the boundary conditions imposed by the specific sample shape. The confinement geometry for the superconducting condensate plays a role similar to that of the confinement potential in the quantum mechanical problem "particle in a box". As a result, the superconducting $H-T$ phase boundary can be changed by choosing the proper sample topology.

\section{RESULTS AND DISCUSSION}

The superconducting $H-T$ phase boundaries are reconstructed by measuring the temperature shift of the midpoint of the normal to superconducting resistive transition at different magnetic fields which were applied perpendicular to the structures. Figure 1 and Figure 2 show the $H-T$ phase boundaries measured in Al samples with different topology. First, we discuss the experimental data for the line. The expected square root behaviour, $H_{c}(T) \propto(I-T / T C)^{1 / 2}$, is convincingly demonstrated (see the solid line in Fig. 1). Secondly, for a mesoscopic loop (Fig. 2) the classical Little-Parks oscillatory $H-T$ phase boundary [1] is observed, which is related to the quantization of the magnetic flux threading the loop area. The oscillation period is $\Delta H=\phi_{0} / S=20.7$ G. Again, the theoretical phase boundary, found by considering the actual sample geometry, describes very well the experimental data (see solid line in Fig. 2).

Finally, Figure 2 also shows the $H-T$ phase diagram of the filled $\mathrm{Al}$ square, which has the same external size and film thickness as the open square. The suppression of the superconducting stafe by the field is much stronger for the filled square, which can easily be explained by the difference of the sample areas penetrated by the magnetic field Moreover, the period $\Delta H$ of the oscillations superimposed on the nearly linear $H_{H}-T$ curve is larger than the period $\Delta H=\phi_{0} / S=20.7 \mathrm{G}$ just expected from the total area $S$ of the filled square.

The main experimental observations for the filled square (Fig. 2) can be summarized as follows: (i) the nearly linear $H-T$ phase boundary, on which the $T_{c}(H)$ oscillations are superimposed, 
is found; (ii) the period of the $T_{c}(H)$ oscillations is different

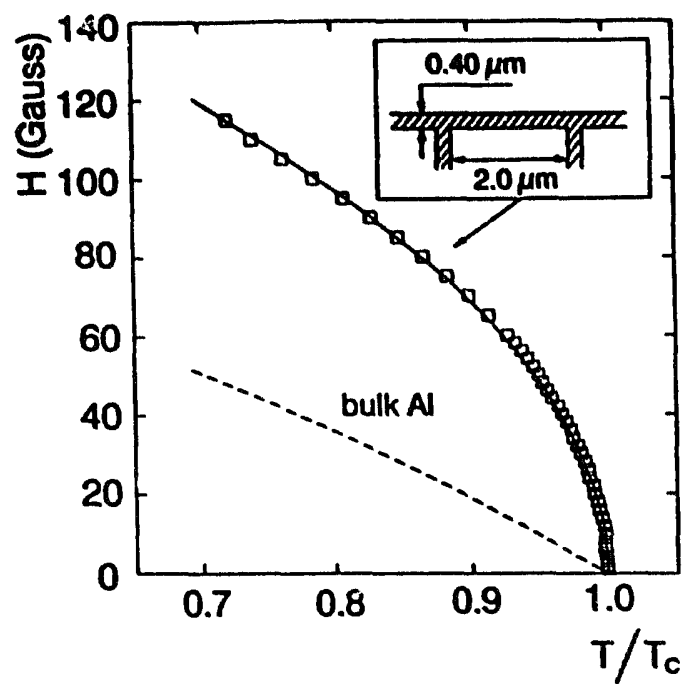

Figure 1. The measured superconducting phase boundary $H_{c}(T)$ as a function of the reduced temperature $T / T_{c}$ for the Al line. The dashed line represents the theoretical calculated phase boundary for bulk $\mathrm{Al}\left(H_{c}(T \rightarrow 0)=100 \mathrm{G}\right)$.

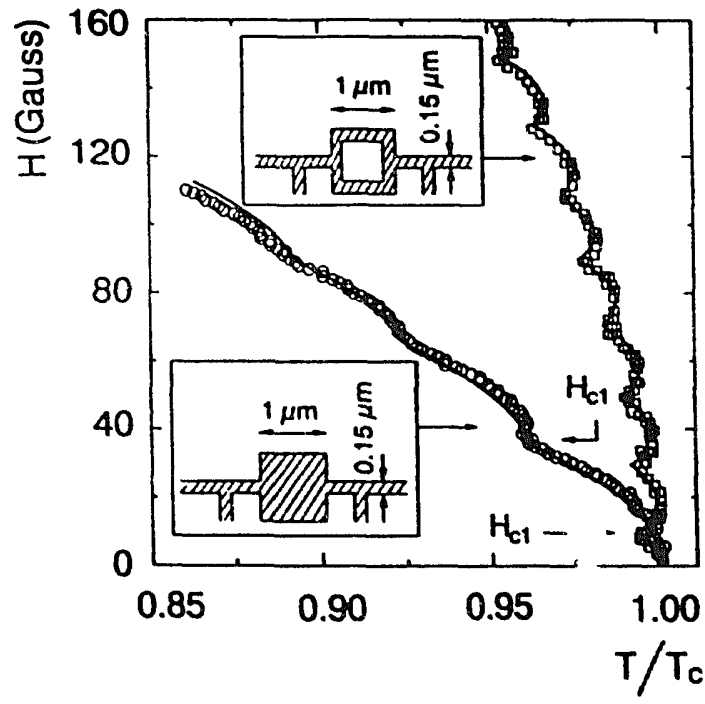

Figure 2. The measured superconducting phase boundary $H_{C}(T)$ as a function of the reduced temperature $T / T_{c}$. The solid lines correspond to calculations (see ref. 2). The lower critical fields $H_{C l}$ correspond to the transition between the states with different orbital quantum number $L$ : from $L=$ 0 to $L=1$. from that of an open square with the same external dimensions; (iki) the first period of the oscillations $\Delta H_{1} \cong 36.4 \mathrm{G}$ is larger than the others $\left(\Delta H_{2} \cong \Delta H_{3} \cong \ldots \cong 27 \mathrm{G}\right.$ ). These observations are fully supported by the theoretical calculations [2].

The analogue of the lower critical field $H_{c 1}$ for mesoscopic structures (Fig. 2) can be found from the transition between the two orbital quantum numbers: $L=0 \rightarrow L=1$. We clearly see again the qualitative difference between $a$ bulk and a mesoscopic superconductor: in the former the $H_{c l}$ value is determined by the material properties, whereas in the latter $H_{C l}$ is strongly dependent upon the sample geometry

\section{CONCLUSION}

Our experiments clearly demonstrate that the superconducting $H-T$ phase boundary in mesoscopic samples is governed by quantization and confinement effects i.e. "quantum design" of the $H-T$ phase boundary line is possible.

\section{ACKNOWLEDGEMENTS}

This research has been supported by the Belgian Inter-University Institute for Nuclear Sciences (IIKW), the Inter-University Attraction Poles (IUAP), and the Concerted Action (GOA) programs. L.G. is Research Assistant of the Belgian Institute for the Encouragement of Scientific Research in Industry and Agriculture (I.W.O.N.L.); C.V.H. is Senior Research Assuciate of the Belgian National Fund for Scientific Research (N.F.W.O.).

\section{REFERENCES}

1. W. A. Little and R. D. Parks, Phys. Rev. Lett. 9 (1962) 9 and R. P.Groff. and R. D. Parks, Phys.

Rev. 176 (1968) 567

2. V. V. Moshchalkov, L. Gielen, C. Strunk, R. Jonckheere, X. Qiu, C. Van Haesendonck and Y. Bruynseraede, submitted to Nature. 